\title{
Experience of Isavuconazole as Salvage Therapy in Chronic Pulmonary Fungal Disease
}

\author{
Lisa Nwankwo ${ }^{*}$ 000-0001-9146-287, Desmond Gilmartin², S Matharu², Ali Nuh ${ }^{3}$, J. Donovan, Darius Arm- \\ strong-James $3,5,6$ and Anand Shah 6,7\$
}

\author{
${ }^{1}$ Pharmacy Department, Royal Brompton Hospital, Guy's and St. Thomas' NHS Foundation Trust, London, \\ SW3 6NP \\ ${ }^{2}$ Clinical Informatics, Royal Brompton and Harefield Hospital Foundation NHS Trust, Fulham, London, SW3 \\ $6 \mathrm{HP}$ \\ ${ }^{3}$ Microbiology Department, Royal Brompton Hospital, Guy's and St. Thomas' NHS Foundation Trust, Lon- \\ don, SW3 6NP \\ ${ }^{4}$ Department of Pathology, Royal Brompton and Harefield NHS Foundation Trust, London, UK, SW3 6NP \\ ${ }^{5}$ MRC Centre for Molecular Bacteriology and Infection, Department of Infectious Diseases, Imperial College \\ London \\ ${ }^{6}$ Department of Respiratory Medicine, Royal Brompton Hospital, Guy's and St. Thomas' NHS Foundation \\ Trust, London, SW3 6NP \\ ${ }^{7}$ MRC Centre of Global Infectious Disease Analysis, Department of Infectious Disease Epidemiology, School of \\ Public Health, Imperial College, London, UK. \\ * Correspondence: 1.nwankwo@rbht.nhs.uk, \\ \$ Second corresponding author: a.shah@imperial.ac.uk
}

\begin{abstract}
Background: The burden of resistant fungal infection is rising in patients with pulmonary disease. Options for antifungal therapy are limited, and the only orally-available antifungals, the triazoles, demonstrate inter and intra-patient variability, non-linear kinetics, toxicity, drug interactions and increasing antifungal resistance. Therapeutic drug monitoring (TDM) of itraconazole, voriconazole and posaconazole has been necessary to ensure their safety and efficacy, but is considered unnecessary for the newest triazole isavuconazole, use of which is increasing. Aims: To characterise isavuconazole susceptibility of Aspergillus fumigatus isolates in a tertiary respiratory referral centre to understand prevalence of isavuconazole antimicrobial resistance. To retrospectively review experience of isavuconazole use in this setting, assessing tolerability and therapeutic drug monitoring. Methods: A retrospective observational analysis of adult patients with respiratory disease in a tertiary hospital setting between Sept 2016 and Aug 2021. Clinical cultures were collected and triazole Minimum inhibitory concentration (MIC) were recorded (based on Clinical \& Laboratory Standards Institute (CLSI method)). Isavuconazole trough drug levels were carried out as part of the standard of care. Clinical outcomes of treatment were evaluated, along with drug tolerance and TDM. Results: During the study period, isavuconazole susceptibility testing was performed on 26 Aspergillus spp isolates. $80.8 \%$ of Aspergillus fumigatus isolates were non-wild type and had isavuconazole MIC > 1mg/L, and 73.0\% had MIC above the EUCAST (European Committee on Antimicrobial Susceptibility Testing) epidemiological cut-off (ECOFF) of $2 \mathrm{mg} / \mathrm{L}$. There was good correlation between isavuconazole MIC and voriconazole MIC ( $\mathrm{r}=0.7, \mathrm{p}=0.0002)$. 54 patients had isavuconazole therapy over the study period with a median duration of 7.7 months (IQR 0.79 16.42). $67 \%$ of patients were able to tolerate isavuconazole, despite toxicity with prior azole treatment being the primary indication for use (in 61.8\%). Increased age ( $\mathrm{r}=0.29 ; \mathrm{p}=0.03(95 \% \mathrm{CI} 0.02,0.52)$ ) and gender ( $\mathrm{r}$ for female sex=-0.31; $\mathrm{p}=0.027$ (95\%CI -0.52,0.036) were associated risk factors for development of adverse events (AEs). 127 Isavuconazole TDM levels were performed over the study period with $90 \%>1 \mathrm{mg} / \mathrm{L}$ and $72 \%>2 \mathrm{mg} / \mathrm{L}$. Dose change from manufacturer's dose recommendation, however, was required in $15 \%$ of patients to achieve a serum drug concentration above the EUCAST ECOFF or Area of technical uncertainty (ATU) value of $2 \mathrm{mg} / \mathrm{L}$. Conclusion: In our study, we show use of Isavuconazole as salvage therapy in chronic pulmonary fungal disease setting with high prevalence of azole resistance. Isavuconazole MICs demonstrated good correlation with voriconazole MICs suggesting the latter could be a useful surrogate marker for isavuconazole susceptibility. Although Isavuconazole achieved excellent serum drug concentrations at standard dose
\end{abstract}


compared to other azole drugs, we highlight the importance of antifungal stewardship and TDM monitoring to optimise therapy in this setting.

Keywords: antifungal resistance; isavuconazole; cystic fibrosis; pulmonary disease; Aspergillus fumigatus; pulmonary aspergillosis; respiratory disease; antifungal stewardship; therapeutic drug monitoring; minimum inhibitory concentration (MIC)

\section{Introduction}

The burden of fungal infection in patients with underlying chronic lung disease is increasing [1]. This is primarily driven by the environmental mould Aspergillus fumigatus, and other filamentous fungi [2]. There is a spectrum of clinical presentation of pulmonary aspergillosis depending on the host immune response and/or the presence of pre-existing lung disease. This ranges from sensitisation and allergic bronchopulmonary aspergillosis (ABPA), to chronic pulmonary aspergillosis (CPA) and invasive fungal disease [3]. CPA occurs in patients with pre-existing lung disease (e.g. mycobacterial disease, chronic obstructive pulmonary disease (COPD), fibrocystic sarcoidosis) and is a slowly destructive lung disease with high mortality, characterised by cavitation, pleural thickening, and raised Aspergillus-specific immunoglobulin G serology [4]. In addition to invasive and semi-invasive disease, ABPA and severe asthma with fungal sensitisation are associated with increased exacerbations and worse outcomes in patients with asthma, bronchiectasis including cystic fibrosis (CF) and to a lesser degree COPD [5]. As such, individuals with fungal infection/sensitisation associated with chronic lung disease often require prolonged antifungal therapy. The global estimate of patients living with chronic pulmonary fungal disease is substantial, with $\sim 5$ million ABPA and $\sim 3$ million CPA individuals respectively $[6,7]$.

Despite the significant burden of chronic respiratory fungal disease, there are only 5 classes of antifungal agents (polyenes, triazoles, echinocandines, pyrimidines and allyamines). The triazoles (itraconazole, voriconazole, posaconazole and isavuconazole) are the only mould-active antifungal drugs available in oral formulation. Prolonged usage is often associated with toxicity and complicated by significant drug-drug interactions and response rates are highly variable. A recent, prospective cohort study in chronic pulmonary aspergillosis showed $\sim 30 \%$ of patients had to stop or change triazole therapy due to toxicity [8].

Therapeutic Drug monitoring (TDM) has been necessary to ensure the safety and efficacy of itraconazole, voriconazole and posaconazole [9-11], but is considered unnecessary for the newest triazole isavuconazole [11], use of which is increasing. TDM is typically necessary where standard dosing gives unpredictable responses, or where there are established dose exposure to response relationships, resulting in either treatment failure, efficacy or toxicity $[9,12]$. In patients with invasive fungal infections (IFIs), sub-therapeutic serum drug concentrations are associated with breakthrough infection or disease progression of IFIs [13]. Within chronic pulmonary fungal disease, we have previously shown a relationship between drug exposure and treatment outcome for ABPA in patients with cystic fibrosis [14]. The additional high prevalence and emergence of antifungal resistance rates seen in chronic pulmonary fungal disease [15-19] alongside high treatment failure rates suggest optimising therapeutic drug exposure may be critical to achieve optimal outcome $[9,10]$.

Itraconazole and voriconazole have been noted to have high inter and intra-patient variability with little or no correlation between dose and plasma level [20-23]. Itraconazole bioavailability can be poor, particularly with capsule formulation [24]. Voriconazole bioavailability is significantly reduced in specific cohorts such as paediatrics, individuals with cystic fibrosis and pancreatic insufficiency and those with CYP2C19 genetic polymorphisms. Posaconazole has improved pharmacodynamic/pharmacokinetic properties 
with the gastro-resistant modified release formulation [25], however antifungal resistance often mirrors that of itraconazole[15], which is prevalent in a chronic pulmonary fungal setting [26]. Drug-drug interactions however remain a significant issue with these triazoles as CYP3A4 inhibitors [27-29]. This is particularly relevant in patients with co-existent mycobacterial infection or CF with the advent of novel CFTR modulator therapies that are metabolized by the cytochrome P450 (CYP) pathway [30].

Isavuconazole is the newest addition to this class of triazole antifungals. Isavuconazole is structurally similar to voriconazole, whilst posaconazole is structurally similar to itraconazole [31]. Isavuconazole received approval in 2015 by the European Medicines Agency (EMA) in Europe and Food and Drug Administration (FDA) in USA [32-34] to treat invasive aspergillosis and invasive mucormycosis in adult patients. It has an advantage of once-a-day dosing, a good pharmacokinetic and pharmacodynamic profile, and lower propensity for drug interactions, however some intra and inter-patient variability has been observed [35, 36]. The implications of reduced drug-drug interactions (DDI) have been shown in a solid-organ transplant population where minimal dose changes to immunosuppressants are required $[37,38]$. Currently it is used in tertiary respiratory practice predominantly as salvage therapy due to toxicity or treatment failure related to alternative azole use [39]. Previous clinical trial data in an invasive fungal disease setting did not demonstrate a relationship between isavuconazole drug exposure and efficacy (clinical response, all-cause mortality) or safety endpoints [40]. To date, there have been no studies of isavuconazole TDM performed in a chronic pulmonary fungal setting where there is increased prevalence of antifungal resistance, drug-drug interactions and cohorts with known reduced bioavailability (e.g. CF) [11].

In this retrospective observational cohort study, we analyse isavuconazole susceptibility of Aspergillus fumigatus isolates in a tertiary respiratory referral centre to understand prevalence of isavuconazole antimicrobial resistance. In addition, we analysed isavuconazole tolerability and therapeutic drug levels. Based on our findings, we propose guidance on therapeutic drug monitoring for isavuconazole use in a chronic respiratory fungal disease setting.

\section{Materials and Methods}

This was a retrospective observational cohort analysis of adult patients at a specialist tertiary respiratory centre over a 5-year period (Sept 2016-Aug 2021). Retrospective electronic health record data collection and protocols were approved by the UK Research Ethics Committee (REC reference:18/HRA/1074). Patients who had either received isavuconazole treatment and/or had isavuconazole drug susceptibility testing on filamentous pathogenic mould isolates were included. Data was obtained from trust electronic prescribing management administration systems following data integration and consequential data mining using SAS Enterprise Guide software. Fungal culture results, isolate antifungal sensitivities, and azole minimum inhibitory concentration (MIC) were recorded, in addition to trough isavuconazole serum levels. Clinical demographics including age, sex, disease-specific information and co-morbidities were collected. Case records were reviewed to analyse drug toxicities (attributed to drug by the clinical team) and reasons for discontinuation or change of azole therapy.

Azole-resistant isolates were confirmed with a standard microbroth dilution method according to Clinical and Laboratory Standards Institute (CLSI) reference guidelines [41]. In our centre, antifungal susceptibility testing for isavuconazole was carried out where requested by the microbiologist/mycologist when resistance to first line therapies (itraconazole/voriconazole) was encountered or where isavuconazole therapy was being considered. Sensitivity testing for the antifungals was determined using the two commercially available antifungal susceptibility testing kits, SensititreTM YeastoneTM (Thermo Fisher) and MICRONAUT-AM (MERLIN Diagnostika) [42]. Isavuconazole is absent from these commercially available antifungal susceptibility testing kits, and thus isavuconazole MIC was determined by the UK Mycology Reference Laboratory in accordance with the 
CLSI broth microdilution method [43]. Isolates with the Epidemiological cut-off (ECOFF) MIC value $>1 \mathrm{mg} / \mathrm{L}$ were considered non-wild type (NWT) (CLSI) [44]. Isavuconazole trough drug levels were carried out as part of the standard of care.

Statistical analysis was performed using GraphPad Prism 9.3.0 software. Tests for normality were performed using Shapiro-Wilk and D'Agostino \& Pearson tests. P values were determined using unpaired $t$ test for parametric variables, and Mann Whitney for non-parametric variables. Pearson's or Spearman's rank correlation tests was used for correlation analysis. A multiple logistic regression analysis was performed to model the probability of adverse effect/no adverse effect. Results were presented as mean \pm standard deviation for parametric variables and median (interquartile range) for non-parametric variables. P-value $<0.05$ was considered significant.

\section{Results}

Within the study period, 850 mould cultures were isolated from respiratory samples in 363 patients. Aspergillus fumigatus was the most predominant isolate at $79.65 \%$. Of these, Isavuconazole sensitivity testing was performed in 29 samples from 21 patients (Supplementary Figure S1).

\subsection{Isavuconazole susceptibility testing}

Within the study period, isavuconazole susceptibility testing was performed on 26 Aspergillus spp isolates. Figure 1 shows the distribution of MIC for Aspergillus fumigatus in the total cohort and also in individuals where Isavuconazole therapy was used.
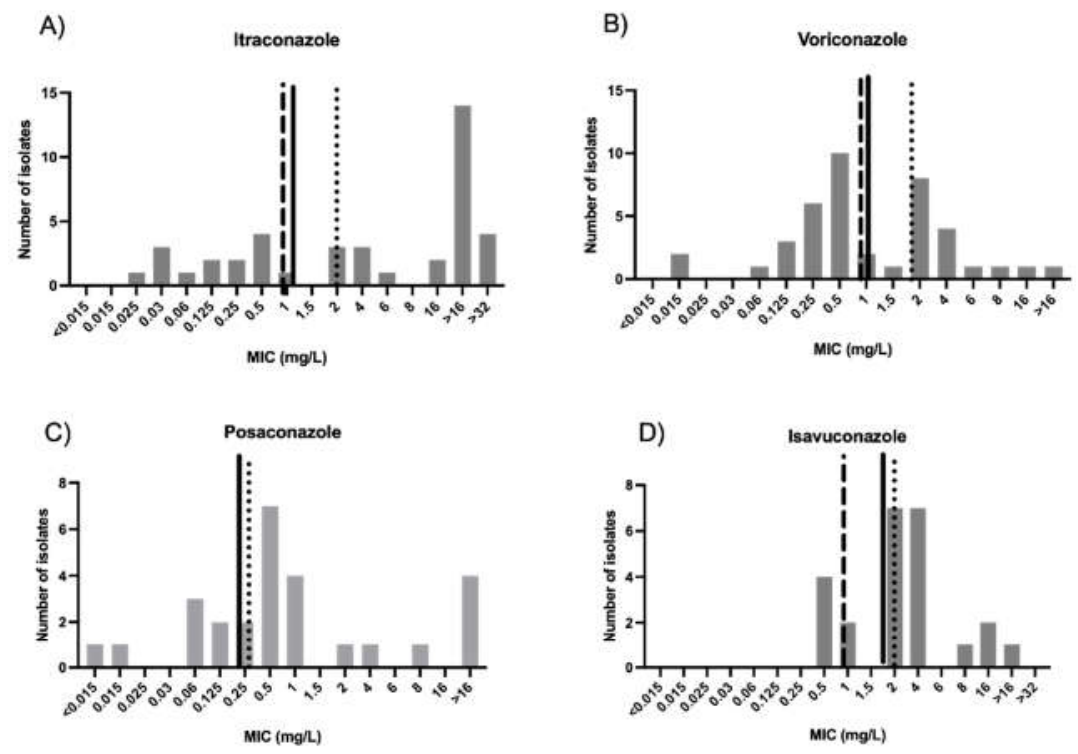

E)

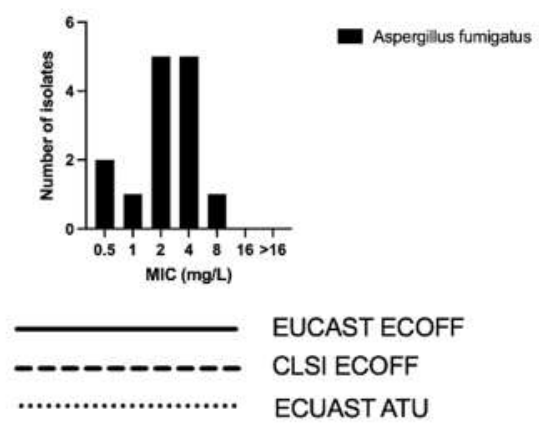

Figure 1. A) Distribution of triazole MIC against all Aspergillus fumigatus clinical isolates for itraconazole during study period; B) Distribution of triazole MIC against all Aspergillus fumigatus clinical isolates for voriconazole during study period; C) Distribution of triazole MIC against Aspergillus fumigatus clinical isolates for posaconazole during study period; D) Distribution of 
triazole MIC against Aspergillus fumigatus clinical isolates for isavuconazole during study period E) Distribution of isavuconazole MIC (mg/L) for Aspergillus fumigatus isolates only in individuals who received isavuconazole during the study period (total 14 isolates). Values higher than the ECOFF value and clinical breakpoint are considered to be resistant. EUCAST=European Committee for Antimicrobial Susceptibility Testing. ECOFF=Epidemiologic cut-off values. ECOFFs are MICs or disk diffusion zone diameters that separate organisms into those "with and without phenotypically detectable resistance". ATU=Area of technical uncertainty

Figure 1 A-D shows the distribution of triazole MIC with the CLSI and EUCAST ECOFF values in all those that received MIC testing. $73 \%$ of isolates had MIC above the EUCAST ECOFF or ATU, and $80.8 \%$ of isolates were above the CLSI ECOFF i.e. non-wildtype for isavuconazole. There was good correlation between Isavuconazole and Voriconazole MIC (Pearson $\mathrm{r}=0.7, \mathrm{p}=0.0002$ (95\%CI 0.43 - 0.85) (Figure 2). MIC levels for nonAspergillus moulds including Lomentospora, Rasamsonia and Exophiala organisms are shown in Supplementary Figure 1

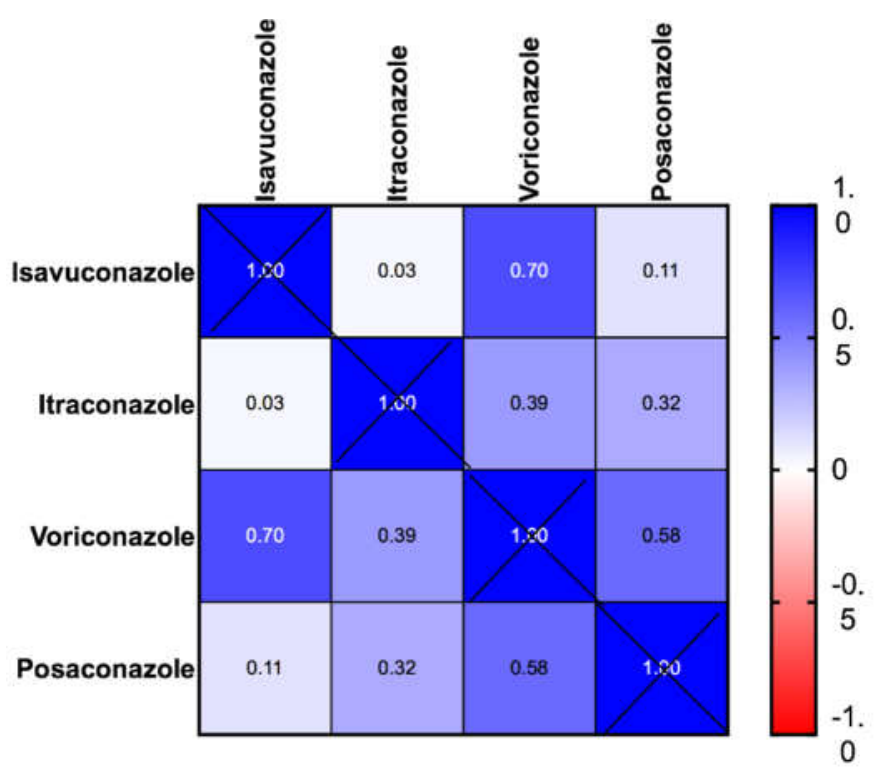

Figure 2. Correlation matrix of triazole MIC (Minimum Inhibitory Concentrations) for all clinical isolates throughout study period.

\subsection{TDM and tolerability of Isavuconazole during study period}

54 patients received isavuconazole therapy over the study period with patient demographics shown in Table 1. Intolerance to other azoles was the predominant reason for isavuconazole use (34 patients, $61.8 \%$ ), with prior alternate azole use high (97\%) given its use as salvage therapy. Isavuconazole was used in 11 patients (20\%) due to treatment failure defined by radiological or serological progression despite alternate azole use (Table $1)$.

Table 1. Patient Demographics; $n=54$.

\begin{tabular}{lc}
\hline Characteristic & No of patients (\%) \\
\hline Age (years) & \\
Median, mean & $56.50,50.52$ \\
(range) & $(21-82)$ \\
\hline
\end{tabular}

$\operatorname{Sex}(\mathrm{N}(\%))$

Male 
Female

Primary Respiratory diagnosis N (\%))

Asthma

$1120.0 \%$

Bronchiolitis obliterans

$1 \quad 1.8 \%$

Cancer

$23.6 \%$

COPD

$35.4 \%$

Cystic Fibrosis

$1933.9 \%$

Immune deficiency

$1 \quad 1.8 \%$

Non-CF bronchiectasis

$5 \quad 9.0 \%$

Post-TB bronchiectasis

$35.4 \%$

NTM

$35.4 \%$

Pulmonary Fibrosis

$11.8 \%$

Sarcoidosis

$7 \quad 12.5 \%$

Pulmonary fungal disease N (\%))

$\begin{array}{lll}\text { ABPA } & 18 & 30.0 \%\end{array}$

Chronic pulmonary aspergillosis

$25 \quad 41.7 \%$

Aspergillus bronchitis

$7 \quad 11.7 \%$

Non-Aspergillus bronchitis

$6 \quad 10.0 \%$

Pulmonary mucormycosis

$1 \quad 1.7 \%$

Aspergillus colonisation

$35.0 \%$

Indication for isavuconazole use (N (\%))

Previous triazole intolerance

$34 \quad 61.8 \%$

Prior antifungal treatment failure

$1120 \%$

Persistently subtherapeutic alternate triazole dosing

$23.7 \%$

Azole resistance

$2 \quad 3.7 \%$

Drug-drug interaction

$23.7 \%$

Better tolerability profile

$35.45 \%$

Mucormycosis oral option (with intolerance of

posaconazole)

$1 \quad 1.81 \%$

Distribution of isolates in patients who received isavuconazole

Fungal species

No of isolates $\mathrm{N}=53(\%)$

Aspergillus fumigatus

$3973.5 \%$

Exophiala dermatitidis

$5 \quad 9.4 \%$

Lomentosprora prolificans

$1 \quad 1.9 \%$

Rasamsonia

$23.8 \%$

Scedosporium apiospermum

$6 \quad 11.3 \%$

ABPA: Allergic Bronchopulmonary Aspergillosis, CF: Cystic Fibrosis, COPD: Chronic Pulmonary aspergillosis, CPA: Chronic Pulmonary Aspergillosis, TB: Tuberculosis, NTM: Non tuberculous Mycobacteria.

Dose variations from standard manufacturer's dose recommendations was seen in $13(24 \%)$ patients. In $5(9.3 \%)$ cases dose escalations in response to lower TDM values or high MIC levels of pathogenic isolates were made, with dose reduction in eight patients $(14.8 \%)$ due to intolerance or supratherapeutic levels. The maximum tolerated dose used 
in this study was $400 \mathrm{mg}$ daily, used in one patient until the patient stopped due to pregnancy. Lower cautious initiation dosing (100mg daily after loading) was used in 11 patients where significant toxicity was seen on previous triazole therapy. Of these, five were still intolerant of isavuconazole, despite the lower than standard initiation dose. Figure 3 shows outcome whilst on isavuconazole treatment during the study period. In $2(5 \%)$ individuals, therapy was stopped due to disease resolution/stability, and $28(52 \%)$ individuals continued with evidence of disease stability. Median treatment duration was 7.73 months (IQR 0.79 - 16.42).

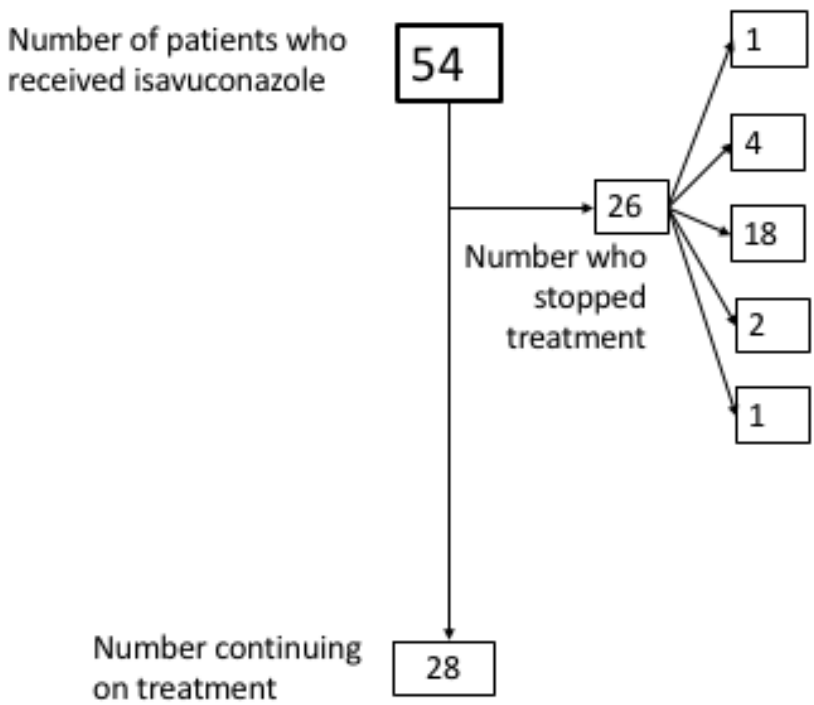

Stopped due to pregnancy, though stable disease

Stopped due to disease progression/death

Stopped due to side effects

Stopped due to disease resolution/stability

Stopped as radiologically responsive and beginning to have side effects

Figure 3. Outcome of individuals treated with isavuconazole for chronic pulmonary fungal disease through the study period $(\mathrm{n}=54)$.

Despite a cohort with very high prior intolerance to azole therapy, isavuconazole treatment was tolerated in $66.6 \%(n=36)$ of patients. Attributable drug toxicity was nevertheless still observed in $33.3 \%$ (18 of 54 patients) (Table 2 ), with hepatotoxicity, skin reactions, and fatigue/drowsiness being the predominant side effect(s).

Table 2. Table of Adverse effects experienced by patients in this study.

\begin{tabular}{|l|l|l|l|l|l|}
\hline $\begin{array}{l}\text { Patient } \\
\text { number }\end{array}$ & Age & Sex & $\begin{array}{l}\text { Treatment } \\
\text { duration In } \\
\text { months }\end{array}$ & $\begin{array}{l}\text { TDM } \\
\text { Isavuconazole } \\
\text { (mean or actual) }\end{array}$ & Toxicity \\
\hline 1 & 27 & M & 0.04 & No TDM available & GI toxicity \\
\hline 2 & 64 & M & 2.36 & 1.97 & Skin reactions, hepatotoxicity \\
\hline 3 & 66 & F & 0.18 & 3.77 & Nausea, headache, insomnia \\
\hline 4 & 20 & F & 10.14 & 3.67 & Dizziness, fatigue \\
\hline 5 & 64 & M & 8.39 & No TDM available & Hepatotoxicity \\
\hline 6 & 60 & F & 2.57 & 1.94 & Headache \\
\hline 7 & 49 & M & 0.25 & No TDM available & Hepatotoxicity \\
\hline 8 & 66 & M & 0.21 & No TDM available & $\begin{array}{l}\text { GI toxicity, taste altered, appetite } \\
\text { decreased, flu-like symptoms }\end{array}$ \\
\hline 9 & 54 & M & 17.46 & 2.48 & Neurotoxicity (delirium) \\
\hline 10 & 67 & F & 1.54 & 3.29 & Drowsiness, skin reactions \\
\hline
\end{tabular}




\begin{tabular}{|l|l|l|l|l|l|}
\hline 11 & 20 & F & 0.071 & No TDM available & Hepatotoxicity \\
\hline 12 & 79 & M & 0.39 & 4.14 & Nausea and vomiting, fatigue \\
\hline 13 & 73 & F & 0.32 & No TDM available & Fatigue \\
\hline 14 & 78 & M & 0.75 & 7.58 & $\begin{array}{l}\text { Facial swelling, difficulty in } \\
\text { micturition, chest discomfort, rectal } \\
\text { mucositis, ankle oedema, breathing } \\
\text { restricted }\end{array}$ \\
\hline 15 & 65 & M & 0.57 & 2.17 & Nausea and vomiting \\
\hline 16 & 82 & F & 1.11 & No TDM available & $\begin{array}{l}\text { Appetite loss, feeling generally } \\
\text { unwell }\end{array}$ \\
\hline 17 & 46 & F & 0.79 & No TDM available & Cardiotoxicity \\
\hline 18 & 62 & M & 15.04 & 4.13 & Skin reactions: Rash \\
\hline
\end{tabular}

Of note, significant hair loss was not observed despite having been a prominent feature in some patients previously on triazole therapy. Increased age $(r=0.29 ; \mathrm{p}=0.03(95 \% \mathrm{CI}$ $0.02,0.52)$ ) and male sex ( $\mathrm{r}$ for female sex=-0.31; $\mathrm{p}=0.027$ (95\%CI -0.52,0.036)) were associated with increased prevalence of adverse effects (Table 3). Therapy was discontinued in only $7.4 \%$ (4 patients) due to disease progression. Six patients died during the study period, of which two were attributed directly to their pre-existing underlying fungal infection with respiratory failure as the cause of death. The other patients died due to complications from their underlying lung disease.

Table 3. Criteria/Risk factors for developing Adverse effect on isavuconazole treatment.

\begin{tabular}{|c|c|c|c|c|}
\hline Criteria/Risk factor & $\begin{array}{c}\text { AEs } \\
\mathrm{N}=\mathbf{1 8}\end{array}$ & $\begin{array}{l}\text { No AEs } \\
\mathrm{N}=34\end{array}$ & $\begin{array}{c}\text { Univariate } \\
\text { p value }\end{array}$ & $\begin{array}{c}95 \% \text { confidence } \\
\text { interval }\end{array}$ \\
\hline \multicolumn{5}{|l|}{${ }^{* *}$ Dose $(\mathrm{mg}), \mathrm{n}$} \\
\hline $100 \mathrm{mg}$ & 6 & 8 & & \\
\hline $200 \mathrm{mg}$ & 14 & 23 & & \\
\hline $300 \mathrm{mg}$ & 0 & 4 & & \\
\hline $400 \mathrm{mg}$ & 0 & 1 & & \\
\hline Age (years) (mean \pm sd) & $58.7 \pm 18.9$ & $46.1 \pm 17.5$ & 0.021 & $0.051,0.537$ \\
\hline \multicolumn{5}{|l|}{ Sex, n (\%) } \\
\hline Male & $10(56 \%)$ & $9(26 \%)$ & & \\
\hline Female & $8(44 \%)$ & $27(74 \%)$ & 0.027 & $-0.527,0.0368$ \\
\hline \multicolumn{5}{|c|}{$\begin{array}{l}\text { Isavuconazole therapeutic } \\
\text { drug level }(\mathrm{mg} / \mathrm{L})\end{array}$} \\
\hline Median (IQR) & $3.19(2.13-3.56)$ & $2.60(1.55-3.85)$ & 0.473 & $-0.200,0.403$ \\
\hline \multicolumn{5}{|c|}{ Duration of therapy (months) } \\
\hline Median (IQR) & $0.57(0.2-2.57)$ & $15.07(9.75-23.89)$ & $<0.0001$ & $-0.795,-0.396$ \\
\hline
\end{tabular}

In individuals who received isavuconazole therapy, Aspergillus fumigatus was the most frequent fungal isolate $(n=39,73.5 \%)$ (Table 1). Other isolates grown included 1 Apergillus niger, 5 Exophiala dermiditidis, 1 Lomentospora prolificans, 2 Rasamsonia argillacea, 1 
Aspergillus terreus, and 6 Scedosporium apiospermum. Where isavuconazole was used as salvage therapy in individuals with chronic lung disease (all with prolonged previous azole exposure), 73.0\% of Aspergillus fumigatus isolates had an isavuconazole MIC above EUCAST ECOFF of $<2 \mathrm{mg} / \mathrm{L}$, and $80.8 \%$ of isolates were $>1 \mathrm{mg} / \mathrm{L}$, the CLSI ECOFF (Fig 1E). Within the study period, $44(81 \%)$ patients treated with isavuconazole had therapeutic trough drug levels performed, with a total of 132 drug levels measured. Of these $86 \%$ (36 patients) attained TDM levels greater than $2 \mathrm{mg} / \mathrm{L}$. Figure 4 shows the distribution of isavuconazole trough levels attained during the study period with a median isavuconazole level of $2.77 \mathrm{mg} / \mathrm{L}$ (IQR 2.35-3.10). 94.8\% of Isavuconazole trough TDM samples were $>1 \mathrm{mg} / \mathrm{L}$, and $72 \%$ of levels $>2 \mathrm{mg} / \mathrm{L}$.

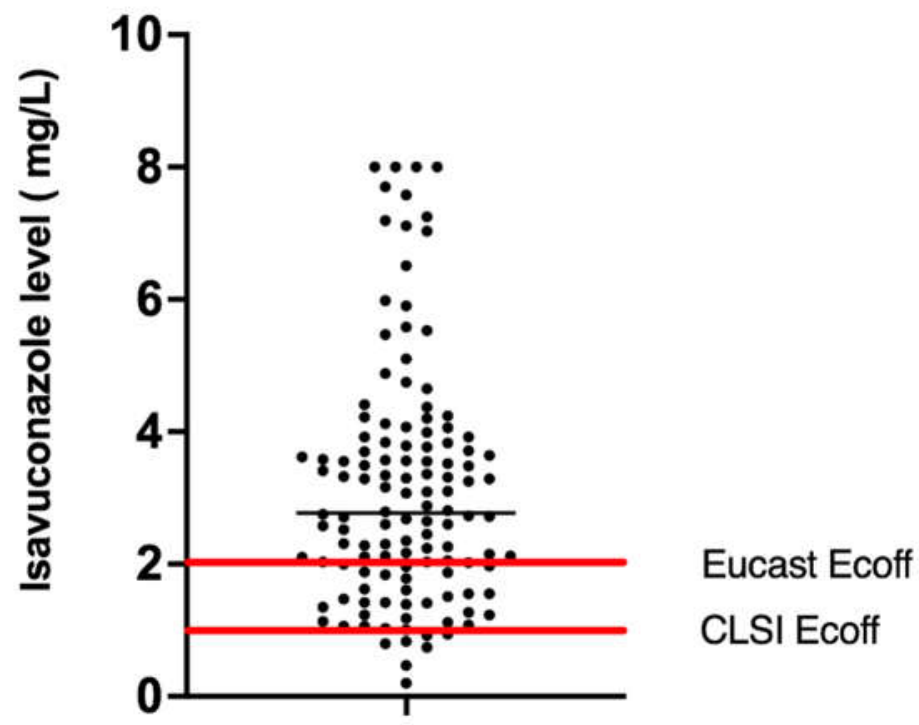

Figure 4. Scatter plot of distribution of isavuconazole drug concentration. The median level is indicated by the horizontal black line, with horizontal red lines indicating the EUCAST and CLSI ECOFF for Aspergillus fumigatus ( $\mathrm{n}=132$ isavuconazole drug level measurements shown).

\section{Discussion}

In this retrospective observational single-centre study, we show use of Isavuconazole across of a range of chronic pulmonary fungal disease with CPA and ABPA being the most common conditions treated. Similar to previous studies, we show a significant degree of azole resistance in chronic respiratory patients with fungal disease [45]. Given the prolonged duration of antifungal therapy required, and the high prior use of azoles in patients receiving isavuconazole as salvage therapy, based on a CLSI ECOFF of $1 \mathrm{mg} / \mathrm{L}$, over $80.8 \%$ of Aspergillus spp. isolates were considered non wild type (resistant to isavuconazole). Given the good correlation of Isavuconazole MIC with voriconazole, our study suggests that voriconazole MIC can confidently be used to screen for isavuconazole sensitivity with resultant beneficial cost implications [46]. Further studies are needed to evaluate application to non-Aspergillus fumigatus species such as Rasamsonia, Scedosporium, and Exophiala sp.

Within our centre, previous azole intolerance was the most common indication for isavuconazole use. Post-marketing surveillance and previous studies have shown high rates of intolerance to first-line azole therapy such as voriconazole and itraconazole [31, 40 ,47]. Despite this, a reasonably high tolerance of isavuconazole was seen in our study $(\sim 66.6 \%)$, suggesting a role even when previous azole intolerance has been encountered. Increasing age was the strongest predictor for isavuconazole AE, with male sex also showing increased AE. Larger studies including pharmacogenomics will be needed to further explore and understand risk factors for azole intolerance which is a significant handicap to prolonged use in chronic fungal disease. Given our results, however, cautious dosing 
may be considered in elderly patients starting isavuconazole, especially if there is a background of previous triazole intolerance, given the good absorption observed during the course of this study.

Of note, significant hair loss requiring treatment interruption was not observed in any of the patients in our study despite having been a feature in a number of patients previously on triazole therapy. Though the exact mechanism of azole mediated hair loss is unclear, voriconazole and itraconazole, have been found to inhibit CYP26A1 mediated hydroxylation of retinoic acid in vitro at concentrations of $>1 \mu \mathrm{M}$ [48], with alopecia reported due to itraconazole, voriconazole and posaconazole. Resultant hair loss due to azoles can cause many patients to stop treatment due to the negative impact on self-esteem, mental health and social interactions. Our results cautiously suggest that in circumstances where patients experience hair loss with itraconazole, voriconazole or posaconazole, isavuconazole may be a good alternative given its reduced CYP inhibition.

A potential advantage of isavuconazole compared to other azoles is its improved pharmacokinetic properties. In our retrospective cohort study, a significant proportion $(86 \%)$ of individuals treated with isavuconazole were able to achieve levels $>2 \mathrm{mg} / \mathrm{L}$ within the study period. This is in contrast to previous studies [49] with high prevalence of subtherapeutic levels where itraconazole, voriconazole and posaconazole were used [9]. Out of the 275 isavuconazole TDM levels taken from this cohort, 67\% were within a target trough level of between $2-5 \mathrm{mg} / \mathrm{L}$ [50]. Supratherapeutic levels were rarely noted and were not associated with an increased propensity towards adverse events. Of note, however, there was a need for dose change from manufacturer's dose recommendations to achieve a drug level above the clinical breakpoint in 15\% of individuals, highlighting the importance of isavuconazole TDM in a chronic respiratory fungal disease setting with high prevalence of antifungal resistance. Increased dosage was well tolerated and not associated with increased adverse events.

At present, isavuconazole is primarily used as salvage therapy. We have however recently shown the high rate of sub-therapeutic azole dosage in a real-world chronic respiratory fungal disease setting, alongside the lack of integrated antifungal stewardship [51]. In this study, we highlight the improved bioavailability and tolerability of Isavuconazole with reduced drug interaction. Further multicentre prospective longitudinal studies are required to understand whether earlier treatment with better tolerated azole antifungal therapy with improved bioavailability, especially in patients with unpredictable bioavailability, would lead to reduced or delayed antifungal resistance acquisition/emergence and improved outcomes.

As a retrospective observational single-centre study, our study has several limitations. Given the absence of isavuconazole from commercially available antifungal susceptibility testing kits, our findings are based on a small number of isavuconazole MIC values, and results need to be replicated in larger multicentre observational chronic pulmonary fungal disease cohort studies. We also need better PK/PD profiling of isavuconazole as in our study there were insufficient data points to perform Monte Carlo simulations and determine a target trough:MIC index, or to evaluate a relationship with this index and efficacy. Given the high rates of antimicrobial resistance seen with salvage isavuconazole therapy, the use of trough:MIC index may be useful to optimise azole dosing. Triazole area under the curve (AUC)/MIC ratio is the standard pharmacodynamic index associated with treatment effect $[52,53]$. However, AUC is impractical to measure in a clinical setting as it requires measurement of drug concentration at a minimum of two timepoints; a $\mathrm{C}_{\min }$ and $\mathrm{C}_{\max }$. Trough:MIC ratios have been explored as a surrogate for AUC/MIC. Exposure/response studies have been carried out with voriconazole using trough:MIC ratio [54], where Monte Carlo simulations determined a trough:MIC ratio of 2 to 5 to be a target for voriconazole therapeutic drug monitoring. Further analysis will be needed to determine what role trough: MIC ratios will play in clinical practice in optimising isavuconazole therapy.

In summary, our study highlights the current use of isavuconazole as salvage therapy in chronic pulmonary fungal disease with high azole resistance prevalence. 
Monitoring of therapeutic drug levels underpins dose optimisation and antifungal stewardship. Isavuconazole MIC demonstrated good correlation with voriconazole MIC and thus the latter can be used as a surrogate marker for isavuconazole susceptibility. Positive treatment outcome or stable disease was seen in majority who tolerated isavuconazole. Isavuconazole is proving to be an important new addition to the repertoire of available antifungals, and shows good bioavailability and tolerability compared to other triazoles used within chronic pulmonary fungal infection. Large multicentre randomised controlled studies are however required to directly assess whether early use of azole therapy with better bioavailability, reduced drug interactions and improved pharmacokinetics can reduce antifungal resistance development in chronic pulmonary fungal disease where prolonged therapy is often required.

Supplementary Materials: The following supporting information can be downloaded at: www.mdpi.com/xxx/s1, Figure S1: Distribution of isavuconazole MIC (x axis, in milligrams per Litre) against Aspergillus spp, Lomentosprora prolificans and Exophiala dermatitidis clinical isolates throughout study period (total 29 clinical isolates)

Author Contributions: Conceptualization, L.N, D.A-J. and A.S.; methodology, L.N, D.A-J. and A.S; validation, L.N, D.A-J. and A.S; formal analysis, L.N, D.A-J. and A.S.; data curation, L.N., S.M. and D.G; writing - original draft preparation, L.N.; writing-review and editing, L.N, AN, D.A-J, A.S. and J.D; Investigation. All authors have read and agreed to the published version of the manuscript.

Funding: LN has received a research fellowship from Royal Brompton Hospital, Guy's and St. Thomas' NHS Foundation Trust, Candidate ID 2019-008. AS is supported by a MRC Clinical Academic Research Partnership award MRC; MR/TOO5572/1 and by the MRC centre grant MRC; MR/R015600/1. DAJ is funded by the Medical Research Council (MR/V037315/1), the Wellcome Trust (219551/Z/19/Z) and the Cystic Fibrosis Trust (SRC015). DAJ is funded by the DHSC Centre for Antimicrobial Optimisation, at Imperial College, London.

Institutional Review Board Statement: The study was conducted in accordance with the Declaration of Helsinki, and approved by the UK Research Ethics Committee (REC reference:18/HRA/1074).

Informed Consent Statement: Patient consent was waived due to the retrospective observational nature of the study.

Data Availability Statement: Not applicable

Acknowledgments: We thank (Chieng Natalie and Soo Jing Wen, pharmacy students) for their assistance with data collection.

Conflicts of Interest: LN reports lecture honoraria from Pfizer, consulting honoraria from Pfizer and MSD, a preceptorship grant from Astellas and travel reimbursements from Pfizer, Basilea, and Astellas. DAJ reports share options in Pulmocide Ltd and speaker fees from Gilead Ltd. AS reports grants from Vertex pharmaceuticals and Gilead Sciences and speaker fees from Pfizer and Gilead Sciences. DG and AN have nil to declare. The funders had no role in the design of the study; in the collection, analyses, or interpretation of data; in the writing of the manuscript, or in the decision to publish the results.

\section{References}

1. Pegorie, M., D.W. Denning, and W. Welfare, Estimating the burden of invasive and serious fungal disease in the United Kingdom. J Infect, 2017. 74(1): p. 60-71.

2. Ohara, S., et al., Clinical characteristics of patients with Aspergillus species isolation from respiratory samples: Comparison of chronic pulmonary aspergillosis and colonization. Respir Investig, 2016. 54(2): p. 92-7.

3. Kousha, M., R. Tadi, and A.O. Soubani, Pulmonary aspergillosis: a clinical review. Eur Respir Rev, 2011. 20(121): p. 156-74.

4. Barac, A., et al., Chronic pulmonary aspergillosis update: A year in review. Med Mycol, 2019. 57(Supplement_2): p. S104-S109.

5. Tiew, P.Y., et al., Environmental fungal sensitisation associates with poorer clinical outcomes in COPD. Eur Respir J, 2020.

6. Bongomin, F., et al., Global and Multi-National Prevalence of Fungal Diseases-Estimate Precision. J Fungi (Basel), 2017. 3(4).

7. Vallabhaneni, S., et al., The Global Burden of Fungal Diseases. Infect Dis Clin North Am, 2016. 30(1): p. 1-11. 
8. Bongomin, F., et al., Twelve-month clinical outcomes of 206 patients with chronic pulmonary aspergillosis. PLoS One, 2018. 13(4): p. e0193732.

9. Laverdiere, M., et al., Therapeutic drug monitoring for triazoles: A needs assessment review and recommendations from a Canadian perspective. Can J Infect Dis Med Microbiol, 2014. 25(6): p. 327-43.

10. Dolton, M.J., et al., Multicenter study of voriconazole pharmacokinetics and therapeutic drug monitoring. Antimicrob Agents Chemother, 2012. 56(9): p. 4793-9.

11. Stott, K.E. and W.W. Hope, Therapeutic drug monitoring for invasive mould infections and disease: pharmacokinetic and pharmacodynamic considerations. J Antimicrob Chemother, 2017. 72(suppl_1): p. i12-i18.

12. Di Paolo, M., et al., A retrospective 'real-world' cohort study of azole therapeutic drug monitoring and evolution of antifungal resistance in cystic fibrosis. JAC Antimicrob Resist, 2021. 3(1): p. dlab026.

13. Dolton, M.J., et al., Multicenter study of posaconazole therapeutic drug monitoring: exposure-response relationship and factors affecting concentration. Antimicrob Agents Chemother, 2012. 56(11): p. 5503-10.

14. Periselneris, J., et al., Posaconazole for the treatment of allergic bronchopulmonary aspergillosis in patients with cystic fibrosis. J Antimicrob Chemother, 2019. 74(6): p. 1701-1703.

15. Abdolrasouli, A., et al., High prevalence of triazole resistance in clinical Aspergillus fumigatus isolates in a specialist cardiothoracic centre. Int J Antimicrob Agents, 2018. 52(5): p. 637-642.

16. Meis, J.F., et al., Clinical implications of globally emerging azole resistance in Aspergillus fumigatus. Philosophical Transactions of the Royal Society B: Biological Sciences, 2016. 371(1709): p. 20150460.

17. Hendrickson, J.A., et al., Antifungal Resistance: a Concerning Trend for the Present and Future. Curr Infect Dis Rep, 2019. 21(12): p. 47.

18. Romero, M., et al., Antifungal Resistance in Clinical Isolates of Aspergillus spp.: When Local Epidemiology Breaks the Norm. J Fungi (Basel), 2019. 5(2).

19. Verweij, P.E., et al., Azole resistance surveillance in Aspergillus fumigatus: beneficial or biased? J Antimicrob Chemother, 2016. 71(8): p. 2079-82.

20. Trifilio, S., et al., Breakthrough fungal infections after allogeneic hematopoietic stem cell transplantation in patients on prophylactic voriconazole. Bone Marrow Transplant, 2007. 40(5): p. 451-6.

21. Ren, Q.X., et al., Population Pharmacokinetics of Voriconazole and Optimization of Dosage Regimens Based on Monte Carlo Simulation in Patients With Liver Cirrhosis. J Pharm Sci, 2019. 108(12): p. 3923-3931.

22. Hope, W.W., Population pharmacokinetics of voriconazole in adults. Antimicrob Agents Chemother, 2012. 56(1): p. 526-31.

23. Scholz, I., et al., Pharmacokinetics, metabolism and bioavailability of the triazole antifungal agent voriconazole in relation to CYP2C19 genotype. Br J Clin Pharmacol, 2009. 68(6): p. 906-15.

24. Grant, E.M., Optimizing the bioavailability of itraconazole. Conn Med, 2000. 64(7): p. 415-7.

25. Lipp, H.P., Clinical pharmacodynamics and pharmacokinetics of the antifungal extended-spectrum triazole posaconazole: an overview. Br J Clin Pharmacol, 2010. 70(4): p. 471-80.

26. Burgel, P.R., et al., High prevalence of azole-resistant Aspergillus fumigatus in adults with cystic fibrosis exposed to itraconazole. Antimicrob Agents Chemother, 2012. 56(2): p. 869-74.

27. Niazi-Ali, S., Pharmacists should make themselves familiar with the antifungal interactions database. The Pharmaceutical Journal, 2020. 305.

28. Peyton, L.R., S. Gallagher, and M. Hashemzadeh, Triazole antifungals: a review. Drugs Today (Barc), 2015. 51(12): p. 705-18.

29. Lipp, H.P., Posaconazole: clinical pharmacokinetics and drug interactions. Mycoses, 2011. 54 Suppl 1: p. $32-8$.

30. Shteinberg, M. and J.L. Taylor-Cousar, Impact of CFTR modulator use on outcomes in people with severe cystic fibrosis lung disease. Eur Respir Rev, 2020. 29(155). 
31. Rybak, J.M., et al., Isavuconazole: Pharmacology, Pharmacodynamics, and Current Clinical Experience with a New Triazole Antifungal Agent. Pharmacotherapy, 2015. 35(11): p. 1037-51.

32. Seyedmousavi, S., P.E. Verweij, and J.W. Mouton, Isavuconazole, a broad-spectrum triazole for the treatment of systemic fungal diseases. Expert Rev Anti Infect Ther, 2015. 13(1): p. 9-27.

33. Ananda-Rajah, M.R. and D. Kontoyiannis, Isavuconazole: a new extended spectrum triazole for invasive mold diseases. Future Microbiol, 2015. 10(5): p. 693-708.

34. Pettit, N.N. and P.L. Carver, Isavuconazole: A New Option for the Management of Invasive Fungal Infections. Ann Pharmacother, 2015. 49(7): p. 825-42.

35. Willeman, T., et al., Refining the therapeutic range of posaconazole and isavuconazole for efficient therapeutic drug monitoring using a bioassay approach. Fundam Clin Pharmacol, 2020. 34(2): p. 279-287.

36. Lewis, R.E., Current concepts in antifungal pharmacology. Mayo Clin Proc, 2011. 86(8): p. 805-17.

37. Kieu, V., et al., Effect of isavuconazole on tacrolimus and sirolimus serum concentrations in allogeneic hematopoietic stem cell transplant patients: A drug-drug interaction study. Transpl Infect Dis, 2019. 21(1): p. e13007.

38. Rivosecchi, R.M., et al., Effects of Isavuconazole on the Plasma Concentrations of Tacrolimus among Solid-Organ Transplant Patients. Antimicrob Agents Chemother, 2017. 61(9).

39. Bongomin, F., et al., Isavuconazole and voriconazole for the treatment of chronic pulmonary aspergillosis: A retrospective comparison of rates of adverse events. Mycoses, 2019. 62(3): p. 217-222.

40. Desai, A., et al., Population Pharmacokinetics of Isavuconazole from Phase 1 and Phase 3 (SECURE) Trials in Adults and Target Attainment in Patients with Invasive Infections Due to Aspergillus and Other Filamentous Fungi. Antimicrob Agents Chemother, 2016. 60(9): p. 5483-91.

41. Chowdhary, A., et al., Comparison of the EUCAST and CLSI Broth Microdilution Methods for Testing Isavuconazole, Posaconazole, and Amphotericin B against Molecularly Identified Mucorales Species. Antimicrob Agents Chemother, 2015. 59(12): p. 7882-7.

42. Nuh, A., et al., Comparative Evaluation of MIRONAUT-AM and CLSI broth microdilution method for antifungal susceptibility testing of Aspergillus species against four commonly used antifungals. Med Mycol, 2022.

43. Borman, A.M., et al., MIC distributions for amphotericin B, fluconazole, itraconazole, voriconazole, flucytosine and anidulafungin and 35 uncommon pathogenic yeast species from the UK determined using the CLSI broth microdilution method. J Antimicrob Chemother, 2020. 75(5): p. 1194-1205.

44. European Committee on Antimicrobial Susceptibility Testing Breakpoint tables for interpretation of MICs for antifungal agents Version 10.0. 2020.

45. Malani, A.N., et al., Alopecia and nail changes associated with voriconazole therapy. Clin Infect Dis, 2014. 59(3): p. e61-5.

46. Gregson, L., et al., In vitro susceptibility of Aspergillus fumigatus to isavuconazole: correlation with itraconazole, voriconazole, and posaconazole. Antimicrob Agents Chemother, 2013. 57(11): p. 5778-80.

47. Eiden, C., et al., Adverse effects of voriconazole: analysis of the French Pharmacovigilance Database. Ann Pharmacother, 2007. 41(5): p. 755-63.

48. Thompson, G.R., 3rd, et al., Examination of Fluconazole-Induced Alopecia in an Animal Model and Human Cohort. Antimicrob Agents Chemother, 2019. 63(2).

49. Di Paolo, M., et al., A retrospective 'real-world' cohort study of azole therapeutic drug monitoring and evolution of antifungal resistance in Cystic Fibrosis. JAC-AMR, 2021. IN PRINT.

50. Risum, M., et al., Therapeutic Drug Monitoring of Isavuconazole: Serum Concentration Variability and Success Rates for Reaching Target in Comparison with Voriconazole. Antibiotics (Basel), 2021. 10(5).

51. Aldossary, S. and A. Shah, Healthcare Utilization and Impact of Antifungal Stewardships Within Respiratory Care Settings: A Systematic Literature Review. Mycopathologia, 2021. 
52. Howard, S.J., et al., Pharmacokinetics and pharmacodynamics of posaconazole for invasive pulmonary aspergillosis: clinical implications for antifungal therapy. J Infect Dis, 2011. 203(9): p. 1324-32.

53. Andes, D., Clinical utility of antifungal pharmacokinetics and pharmacodynamics. Curr Opin Infect Dis, 2004. 17(6): p. 533-40.

54. Troke, P.F., H.P. Hockey, and W.W. Hope, Observational study of the clinical efficacy of voriconazole and its relationship to plasma concentrations in patients. Antimicrob Agents Chemother, 2011. 55(10): p. 4782-8.

Supplementary figure

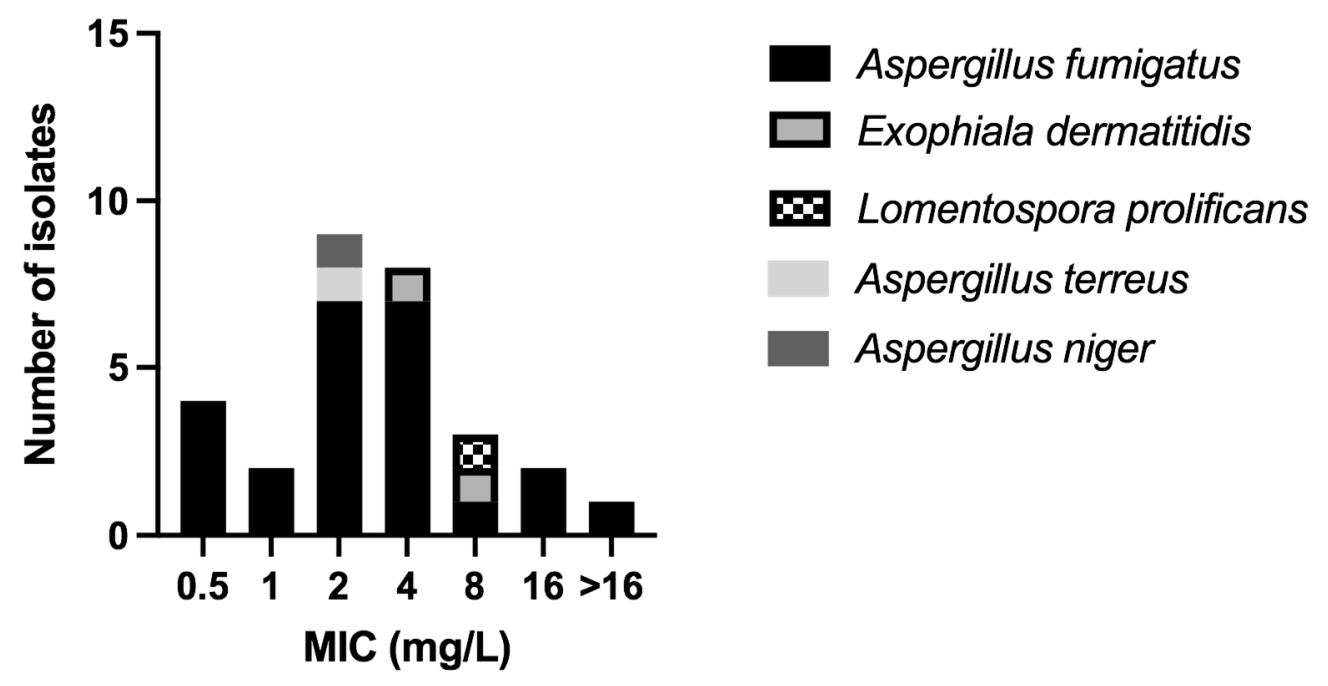

Figure S1: Distribution of isavuconazole MIC (x axis, in milligrams per Litre) against Aspergillus spp, Lomentosprora prolificans and Exophiala dermatitidis clinical isolates throughout study period (total 29 clinical isolates) 